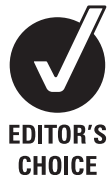

CHOICE

\title{
Blind subjects implanted with the Argus II retinal prosthesis are able to improve performance in a spatial-motor task
}

\author{
A K Ahuja, ${ }_{1}^{1}$ J D Dorn, ${ }^{1}$ A Caspi, ${ }^{1}$ M J McMahon, ${ }^{1}$ G Dagnelie, ${ }^{2}$ L daCruz, ${ }^{3}$ \\ P Stanga, ${ }^{4}$ M S Humayun, ${ }^{5}$ R J Greenberg, ${ }^{1}$ Argus II Study Group
}

${ }^{1}$ Second Sight Medical Products, Sylmar, California, USA

${ }^{2}$ Lions Vision Research and Rehab Center, Baltimore, Maryland, USA

${ }^{3}$ Moorfields Eye Hospital, London, UK

${ }^{4}$ Manchester Royal Eye Hospital, Manchester, UK ${ }^{5}$ Dohey Eye Institute, Keck School of Medicine, Los Angeles, California, USA

Correspondence to Dr Ashish K Ahuja, Sight Medical Products, bldg. 3 12744 San Fernando Blvd Sylmar, CA 91342, USA; aahuja@2-sight.com

Accepted 24 May 2010 Published Online First 29 September 2010

\begin{abstract}
Background/aims To determine to what extent subjects implanted with the Argus II retinal prosthesis can improve performance compared with residual native vision in a spatial-motor task.
\end{abstract}

Methods High-contrast square stimuli (5.85 cm sides) were displayed in random locations on a $19^{\prime \prime}(48.3 \mathrm{~cm})$ touch screen monitor located $12^{\prime \prime}(30.5 \mathrm{~cm})$ in front of the subject. Subjects were instructed to locate and touch the square centre with the system on and then off (40 trials each). The coordinates of the square centre and location touched were recorded.

Results Ninety-six percent (26/27) of subjects showed a significant improvement in accuracy and 93\% (25/27) show a significant improvement in repeatability with the system on compared with off ( $p<0.05$, Student $t$ test). A group of five subjects that had both accuracy and repeatability values $<250$ pixels $(7.4 \mathrm{~cm})$ with the system off (ie, using only their residual vision) was significantly more accurate and repeatable than the remainder of the cohort $(p<0.01)$. Of this group, four subjects showed a significant improvement in both accuracy and repeatability with the system on.

Conclusion In a study on the largest cohort of visual prosthesis recipients to date, we found that artificial vision augments information from existing vision in a spatial-motor task.

Clinical trials registry no NCT00407602.

\section{INTRODUCTION}

Second Sight Medical Products (Sylmar, California, USA) has developed an epiretinal prosthesis aimed at partially restoring vision to people blinded by outer retinal degenerative diseases such as retinitis pigmentosa (RP). In these diseases, while the photoreceptors are compromised and there is anatomical remodelling of the remnant retina, ${ }^{1}{ }^{2}$ post mortem anatomical studies have found that some bipolar and ganglion cells survive. ${ }^{3}{ }^{4}$ Multiple acute and chronic studies in normal ${ }^{5-9}$ and degenerate $^{10}$ animal models, and in human subjects, have shown that electrical stimulation of the retina can elicit percepts (phosphenes). ${ }^{11-13}$ More recently it has been demonstrated that stimulation with multiple electrodes can yield some level of spatial vision as measured by high-contrast square-wave grating tests. $^{14}$

The Argus II retinal prosthesis system (Second Sight Medical Products) consists of a surgically implanted 60-electrode stimulating microelectrode array consisting of $200 \mu \mathrm{m}$ diameter disc electrodes, an inductive coil link used to transmit power and data to the internal portion of the implant, an external belt-worn video processing unit (VPU) and a miniature camera mounted on a pair of glasses (figure 1). The video camera captures a portion of the visual field and relays the information to the VPU. The VPU digitises the signal in real-time, applies a series of image processing filters, downsamples the image to a $6 \times 10$ pixelised grid, and creates a series of stimulus pulses based on pixel brightness values and look-up tables customised for each subject. The stimulus pulses are delivered to the microelectrode array via application-specific circuitry and a superior-temporally placed inductive radio frequency coil link allowing for wireless forward and reverse telemetry between intra and extra-ocular portions of the system.

Here we present results of a task requiring the integration of visual information afforded by the Argus II retinal prosthesis with motor movementthe localisation of a high contrast square target on a computer monitor.

\section{METHODS}

Blind subjects with severe to profound RP were implanted with the Argus II prosthesis as part of a clinical study at multiple clinical sites worldwide. Those centres were: Doheny Eye Institute at the University of Southern California (Los Angeles, California, USA); Retina Foundation of the Southwest (Dallas, Texas, USA); Moorfields Eye Hospital (London, UK); University of California at San Francisco (San Francisco, California, USA); Wilmer Eye Institute at the Johns Hopkins School of Medicine (Baltimore, Maryland, USA); Wills Eye Institute at the University of Pennsylvania (Philadelphia, Pennsylvania, USA), the Edward S. Harkness Eye Institute at Columbia University (New York, USA); Puerta de Hierro Centro Medico (Guadalajara, Mexico); Centre Hospitalier National d'Ophtalmologie des Quinze-Vingts (Paris, France); Hôpitaux Universitaires de Genève (Geneva, Switzerland). All data presented here were collected at these clinical sites, with the exception of sites in Philadelphia and New York, where psychophysical testing and data collection were done at the Scheie Eye Institute at the University of Pennsylvania and Lighthouse International, respectively.

All implanted subjects had some level of bare light perception (BLP) prior to surgery, and at the time of the latest clinical follow-up. No subjects had a recordable visual acuity prior to surgery (worse than 2.9 logarithm of the minimum angle of 
Figure 1 A schematic illustration showing the surgically implanted stimulating microelectrode array, and inductive coil telemetry link of the Argus II system (left). The external portions of the system consist of a video processing unit (VPU) (middle) and a miniature camera mounted on a pair of glasses (right).
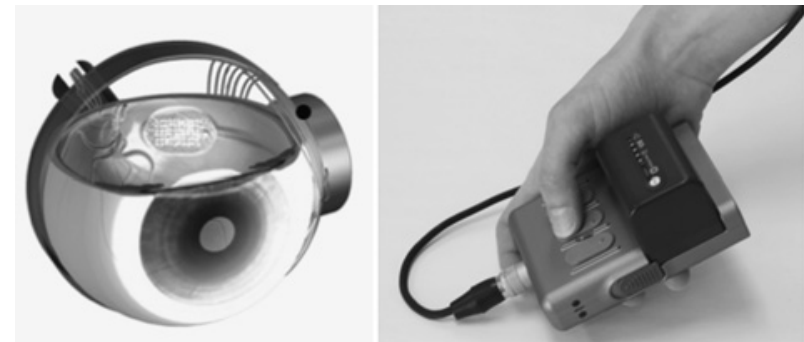

resolution (logMAR)) (ie, worse than 20/15887) as determined by an adaptive four alternative force choice (4-AFC) square wave grating test.

The experiments described in here were run on all Argus II subjects who were currently available for regular testing and who had been implanted before 1 July 2009 (27 subjects). Nineteen of the 27 subjects $(70 \%)$ included in this study were male. At the time of publication the mean subject age was $58 \pm 10$ (range 27-77) years; the mean duration of implantation

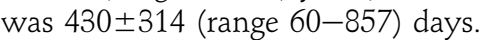

In the experiment, white square stimuli $\left(5.85 \mathrm{~cm}^{2}, 200\right.$ pixels ${ }^{2}$, $221 \mathrm{~cd} / \mathrm{m}^{2}$ ) were displayed in random locations on a $19^{\prime \prime}$ $(48.3 \mathrm{~cm}$ ) LCD touch screen monitor (ELO Accutouch or IntelliTouch Surface Wave LCD Touch Screen Flat Monitor 1915L; Menlo Park, California, USA) located $12^{\prime \prime}(30.5 \mathrm{~cm})$ in front of the subject (subtending a visual angle of $10.9^{\circ}$ ). After the onset of the square and an auditory prompt, the subjects were instructed to locate and touch the square centre. The program provided verbal feedback after subject's responses indicating 'correct' for a touch anywhere on the square and 'close' for a touch within 100 pixels $(2.9 \mathrm{~cm})$ of the edge of the square. Corrective feedback was given after 'close' and incorrect responses. For example, a response $6 \mathrm{~cm}$ to the left of and $4 \mathrm{~cm}$ below the stimulus square prompted the response 'It was higher and right.'

Before the test was administered, 10 trials were presented in 'training mode' to familiarise the subject with the position and vertical and horizontal extent of the monitor. In this mode subjects defined the location of the square centre by pressing the blank touch screen monitor; a $5.85 \mathrm{~cm}$ square appeared at that location until subjects touched another part of the monitor. The subjects' cameras were aligned with respect to the monitor centre. Experiments consisting of 40 trials were run with both the system on and off (80 trials total per subject). The subjects' eyes were not patched in either condition; in system off testing the subjects did not wear the Argus II system glasses.

Subjects were instructed to move their head while keeping their eyes centred relative to their head position. (Since the stimulating array is fixed on retina, the perceived phosphene location depends on eye and head position.) The $x-y$ pixel coordinates of the square centre and of the location touched were recorded for off-line analysis and plotting using Matlab 7.1, Microsoft Excel 2007 and Igor Pro 6.1.

\section{RESULTS}

The accuracy of subject responses in an experiment was determined by calculating the mean distance between individual responses and target square centre. For display purposes, subjects were ordered from smallest improvement in accuracy with the system on compared with off, to largest improvement system on compared with off. The ordered list of 27 subjects was divided into nine sections, and system on and system off results for the median performer from each sub-section are shown in figure 2. In these plots the responses are plotted relative to a fixed target square location centred at $(0,0)$. These nine subjects are representative of the range of results.

Each subject's mean accuracy for system on and off is presented in figure 3. (Subjects showing the greatest improvement with the system on are presented on the right.) Ninety-six percent (26/27) of subjects showed statistically significant improvement in accuracy with the system on compared with off $(p<0.05$, two-tailed unpaired Student t test). Subject JHU-003 was slightly more accurate with the system on compared with off; however, the improvement was not statistically significant. The mean distance from the target for all subjects for all tests was $149 \pm 50$ pixels with the system on, and $323 \pm 94$ pixels with the system off.

The repeatability (or clustering) of subject's responses was determined by calculating the mean difference between individual responses and the mean of all responses. Ninety-three percent $(25 / 27)$ of subjects have significantly better repeatability with the system on compared with off. (JHU-003 and JHU-005 are more repeatable with the system off compared with on.) There is a high correlation between subjects' repeatability and their accuracy with both the system on $\left(R^{2}=0.88\right.$, slope $\left.=0.82\right)$ and off $\left(R^{2}=0.90\right.$, slope $\left.=1.00\right)$ (figure 4$)$.

\section{DISCUSSION}

The square localisation task described here assessed the ability of blind subjects implanted with the Argus II retinal prosthesis system to localise and touch a high contrast square target on a touch screen monitor. Successful performance of the task required subjects to develop adequate head-scanning technique and good 'camera-hand' coordination. Ninety-six percent of implanted subjects studied had responses that were significantly more accurate, and 93\% had responses that were more repeatable with the system on compared with the system off. There was a high correlation between accuracy and repeatability with both the system on $\left(R^{2}=0.88\right)$ and off $\left(R^{2}=0.90\right)$. This is intuitive since a subject that can more often correctly determine the target centre also gets more visual feedback with which to refine motor movement.

For the subjects who showed significant improvement in accuracy with the system on compared with the system off, the factor of improvement ranged from 1.25 (LON-001) to 4.63 (JHU-002). This range was largely due to the variability in performance with the system off. (The standard deviation of the mean accuracy across all subjects was 86.3 pixels with the system off compared with 51.4 with the system on.) In other words, the rare cases of only marginal improvement in accuracy with the system on were due to the fact that a few subjects had enough light perception and eye-hand coordination to perform the task with their native vision. This limited the possible range of improvement with the system on. Possible secondary explanations for the wide range of factor of improvement include differences in array-retina apposition and array-macula 


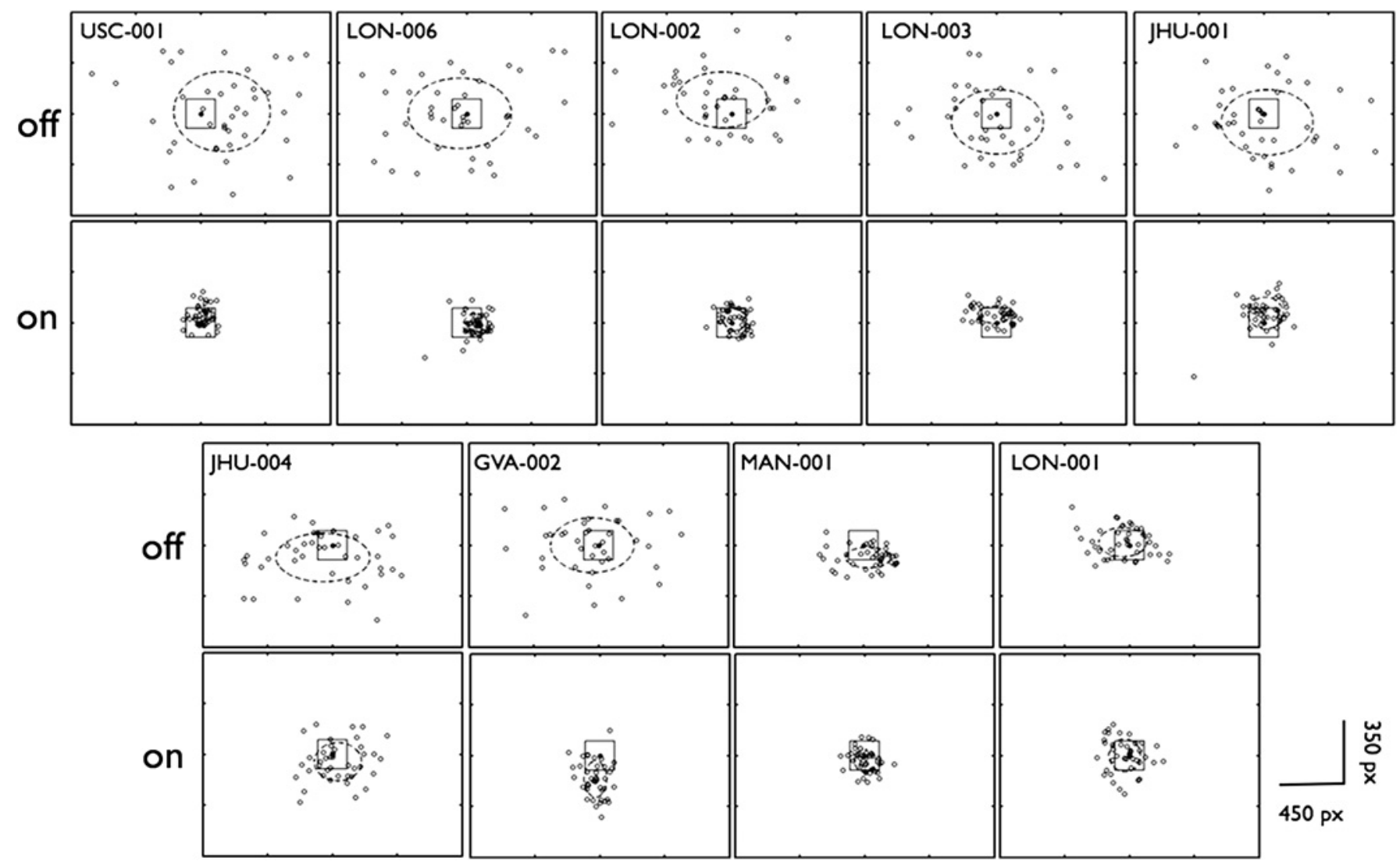

Figure 2 Results from square localisation experiments of nine representative subjects with the system off and on. Plots are normalised to square centre; responses (white circles) are plotted relative to target square location (axes units are in pixels). Standard deviations of the mean of all responses in an experiment are indicated (dotted line). The subjects are plotted from smallest (top left) to largest (bottom right) factor of improvement in accuracy (system on vs off).

distance, and in subject-specific variability in performing psychophysical tasks.

Case reports of those whose sight is partially restored after years of blindness indicate that motor tasks requiring hand-eye coordination (eg, maze tracing, picking up small pellets) are adversely affected after prolonged binocular visual deprivation. ${ }^{15}{ }^{16}$ Similarly, subjects in this study who had the most success on this task with their native vision were still
Figure 3 Mean distance from target square centre (pixels) for each subject with system on (black square) and off (white circles). Range of standard error of mean is shown by vertical bars. Subjects are plotted in order of increasing factor of improvement in accuracy (system on vs off).

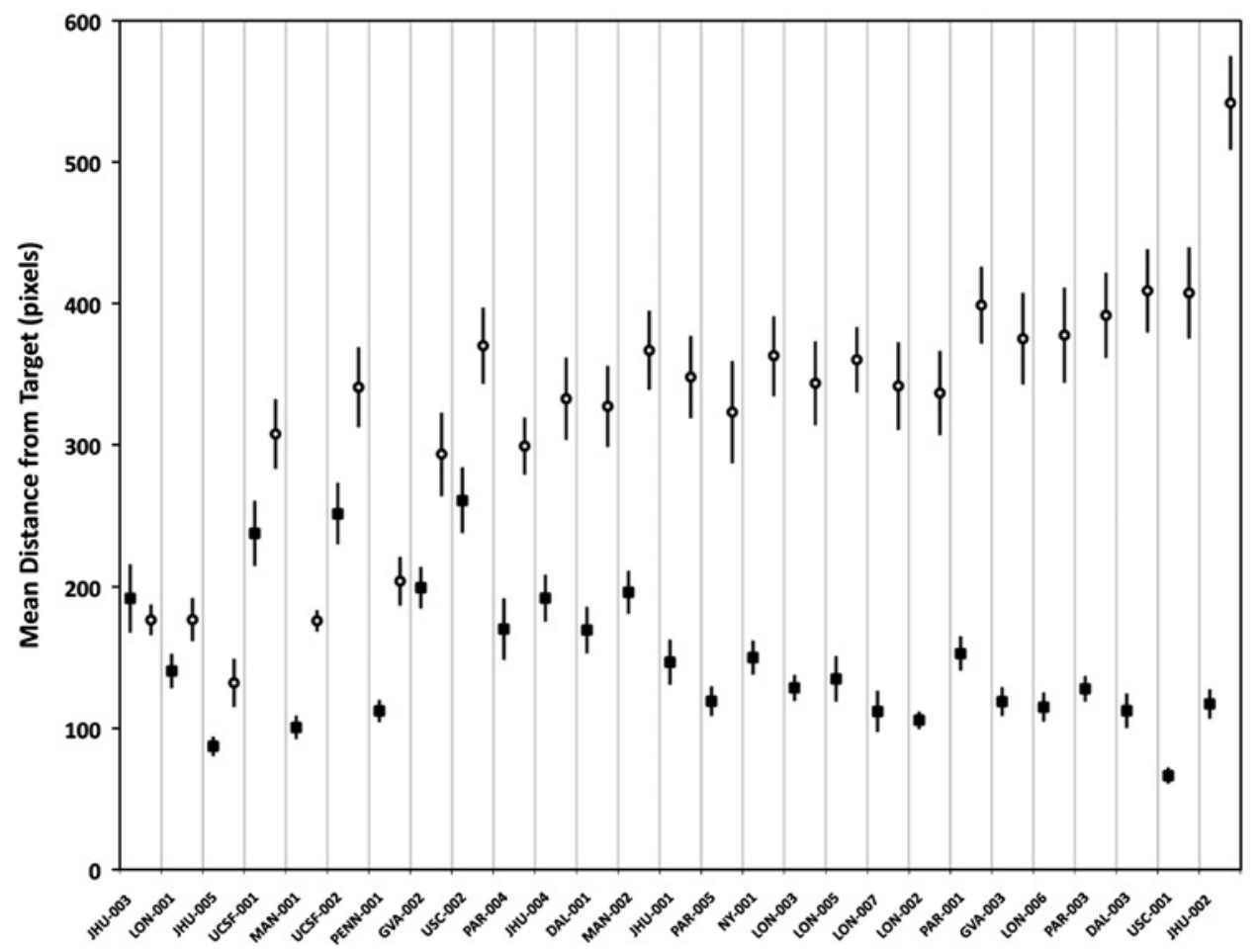




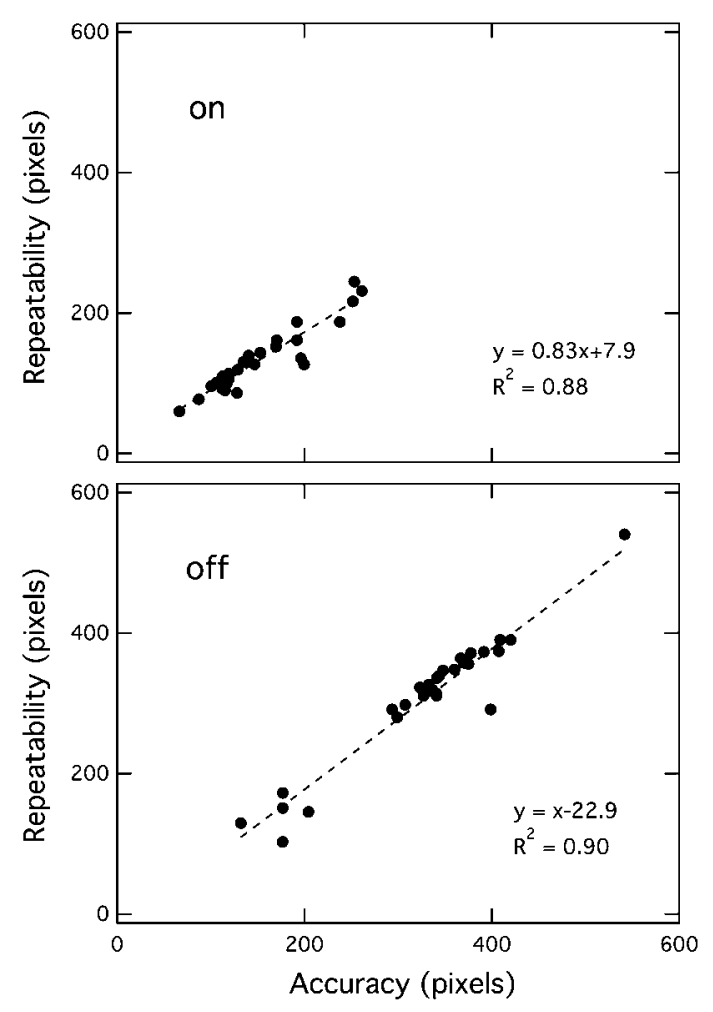

Figure 4 Repeatability (pixels) versus accuracy (pixels) for all subjects with system on (top) and system off (bottom). Linear regression (dashed line) for system off $\left(y=1.00 x-22.9 ; R^{2}=0.90\right)$ and system on $(y=0.82 x+$ 7.9; $R^{2}=0.88$ ) shows a good correlation between subject's ability mean response distance from target and the clustering of their responses. The five subjects (JHU-003, LON-001, JHU-005, MAN-001 and PENN-001) that had success with the task with their native vision are clearly separated from the rest of the cohort in the system off plot.

consistently off the square centre with system off due to years of lack of visual feedback (JHU-003, MAN-001, PENN-001; figure 5). Therefore, in determining subjects who could perform the task with their native vision, both accuracy and repeatability were considered. The bottom of figure 4 shows a plot of repeatability versus accuracy with system off. There is a clear clustering of five subjects who were significantly better at performing the task with their native vision compared with all other subjects. These subjects (JHU-003, LON-001, JHU-005, MAN-001 and PENN-001) had both repeatability and accuracy values $<250$ pixels. Even though these subjects had enough residual native vision and visual-motor feedback to perform well, remarkably four of five of these subjects still showed a significant improvement in accuracy with system on compared with off and had a mean distance from the square centre of less than 150 pixels $(4.4 \mathrm{~cm}$ ) with the system on (all except JHU-003). This suggests that these subjects were able to synergise visual input from the prosthesis system with their residual native vision, which would be a prerequisite for implanting individuals with better sight with the Argus II system. This perhaps surprising result that artificial vision augmented performance rather than interfered with it lends support to the idea that, like with other sensory prostheses such as cochlear implants, individuals with better vision than those studied here may also benefit from prosthesis use. We believe this is the first report of a visual prosthesis improving the performance of blind subjects, even those with a measureable

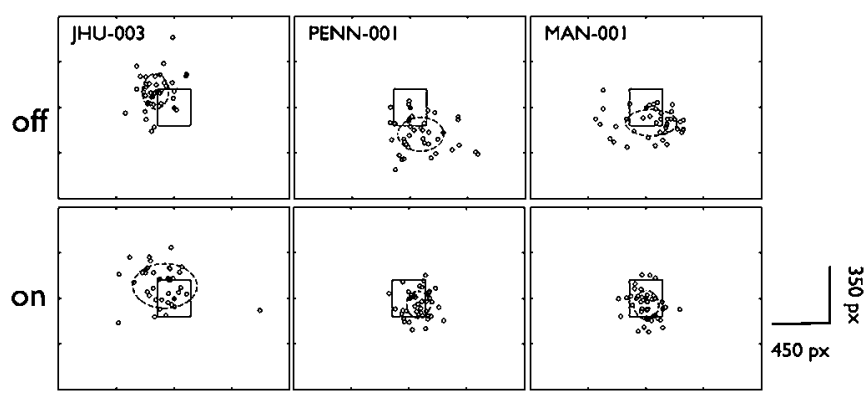

Figure 5 Results from square localisation experiments with the system off (top) and on (bottom) for JHU-001, MAN-001 and PENN-001. Plots are normalised to square centre; responses (empty circles) are plotted relative to target square location (axes units are in pixels). Standard deviations of the mean of all responses in an experiment are indicated (dotted line). Although all subjects could perform the task with their native vision, years of visual deprivation effect of spatial-motor feedback. Two of three (MAN-001 and PENN-001) of these subjects' accuracy and repeatability improved with the system on.

amount of native vision, in a spatial-motor task over such a large cohort.

Funding National Institutes of Health (Bethesda, MD, USA) grant no. 5R01EY12893.

Competing interests AKA, JDD, AC, MJMcM and RJG are employees of and have a financial interest in Second Sight Medical Products. GD, LdaC and PS belong to institutions that receive funding from Second Sight Medical Products to support the study. The individuals themselves have no financial interest in Second Sight Medical Products.

\section{Patient consent Obtained.}

Ethics approval This study was conducted with ethics approval from all participating clinical sites: Doheny Eye Institute at the University of Southern California (Los Angeles, California, USA); Retina Foundation of the Southwest (Dallas, Texas, USA); Moorfields Eye Hospital (London, UK); the University of California at San Francisco (San Francisco, California, USA); Wilmer Eye Institute at the Johns Hopkins School of Medicine (Baltimore, Maryland, USA): Wills Eye Institute at the University of Pennsylvania and the Scheie Eye Institute (Philadelphia, Pennsylvania, USA), The Edward S. Harkness Eye Institute at Columbia University and Lighthouse International (New York, USA); Puerta de Hierro Centro Medico (Guadalajara, Mexico); Centre Hospitalier National de Ophtalmologie des Quinze-Vingts (Paris, France); Hôpitaux Universitaires de Genève (Geneva, Switzerland).

Provenance and peer review Not commissioned; externally peer reviewed.

\section{REFERENCES}

1. Marc RE, Jones BW, Watt CB, et al. Neural remodeling in retinal degeneration. Prog Retin Eye Res 2003;22:607-55.

2. Jones BW, Marc RE. Retinal remodeling during retinal degeneration. Exp Eye Res 2005:81:123-37.

3. Santos A, Humayun MS, de Juan E Jr, et al. Preservation of the inner retina in retinitis pigmentosa. a morphometric analysis. Arch Ophthalmol 1997;115 $511-15$.

4. Stone JL, Barlow WE, Humayun MS, et al. Morphometric analysis of macular photoreceptors and ganglion cells in retinas with retinitis pigmentosa. Arch Ophthalmol 1992:110:1634-9.

5. Jensen RJ, Ziv OR, Rizzo JF 3rd, et al. Spatiotemporal aspects of pulsed electrical stimuli on the responses of rabbit retinal ganglion cells. Exp Eye Res 2009;89:972-9.

6. Greenberg. Visual prosthesis and related technology 2001:4:1-16.

7. Behrend M, Ahuja A, Humayun MS, et al. Electrical stimulation of the retina in a 3-dimensional interface. In: Fort Lauderdale, FL: Conference proceedings of ARVO, 2009.

8. Ahuja AK, Behrend MR, Kuroda M, et al. An in vitro model of a retinal prosthesis. IEEE Trans Biomed Eng 2008;55:1744-53.

9. Crapper DR, Noell WK. Retinal excitation and inhibition from direct electrical stimulation. J Neurophysiol 1963;26:924-47.

10. Sekirnjak C, Hulse C, Jepson LH, et al. Loss of responses to visual but not electrical stimulation in ganglion cells of rats with severe photoreceptor degeneration. J Neurophysiol 2009;102:3260-9. 
11. Rizzo JF 3rd, Wyatt J, Loewenstein J, et al. Perceptual efficacy of electrical stimulation of human retina with a microelectrode array during short-term surgical trials. Invest Ophthalmol Vis Sci 2003;44:5362-9.

12. Humayun MS, de Juan $\mathrm{E} \mathrm{Jr}$, Weiland JD, et al. Pattern electrical stimulation of the human retina. Vision Res 1999;39:2569-76.

13. Mahadevappa M, Weiland JD, Yanai D, et al. Perceptual thresholds and electrode impedance in three retinal prosthesis subjects. IEEE Trans Neural Syst Rehabil Eng 2005;13:201-6
14. Caspi A, Dorn JD, McClure $\mathrm{KH}$ et al. Feasibility study of a retinal prosthesis: spatial vision with a 16-electrode implant. Arch Ophthalmol 2009;127:398-401.

15. Carlson S, Hyvarinen L, Raninen A. Persistent behavioural blindness after early visua deprivation and active visual rehabilitation: a case report. $\mathrm{Br} J$ Ophthalmol 1986;70:607-11.

16. Ostrovsky Y, Andalman A, Sinha P. Vision following extended congenital blindness Psychol Sci 2006;17:1009-14.

\section{Have confidence in your decision making.}
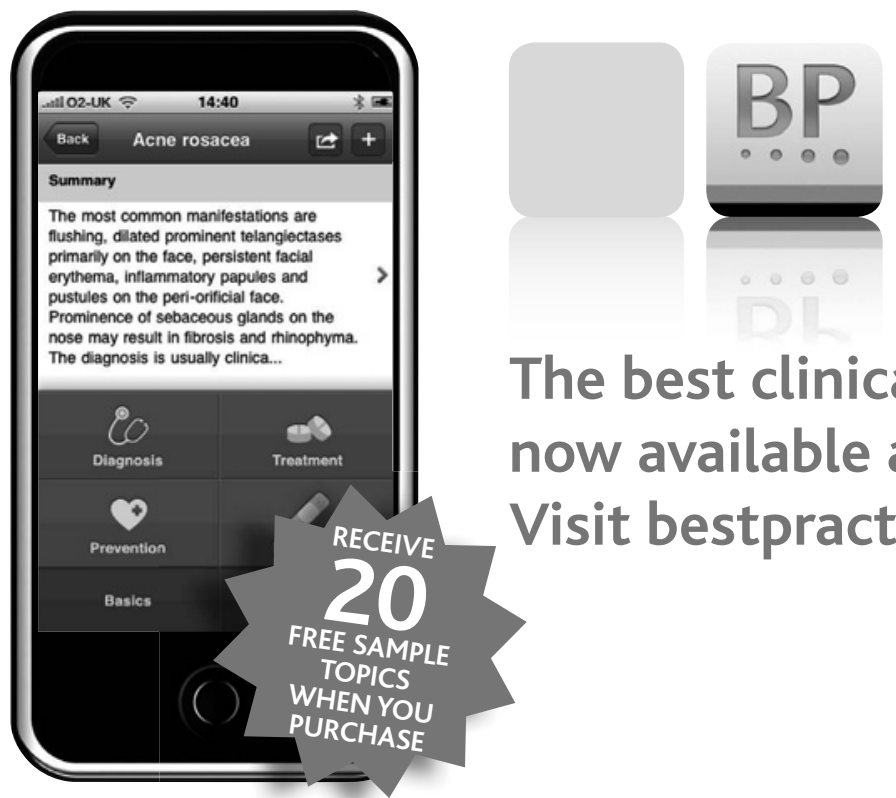

The best clinical decision support tool is now available as an app for your iPhone. Visit bestpractice.bmj.com/app

\section{clinicians $\bullet$ medical students $\bullet$ nurses $\bullet$ healthcare practitioners}

